\title{
Endocervical adenocarcinoma in situ with ovarian metastasis: a
}

\section{case report}

\author{
Špela Smrkolj $^{1,2}$, Snježana Frković Grazio ${ }^{3}$, Renata Košir Pogačnik ${ }^{1}$ \\ ${ }^{1}$ Division of Gynecology and Obstetrics, University Medical Centre Ljubljana, Ljubljana, Slovenia \\ ${ }^{2}$ Faculty of Medicine; University of Ljubljana, Ljubljana, Slovenia \\ ${ }^{3}$ Division of Gynecology and Obstetrics, Department of Gynecologic Pathology, University Medical Centre Ljubljana, Ljubljana, Slovenia
}

\begin{abstract}
Summary
Background: Ovarian metastases, which may simulate primary ovarian neoplasms, can occur in advanced stage primary cervical adenocarcinoma (AC). However, such tumors are very rarely observed in cases of endocervical adenocarcinoma in situ (AIS) with early or even no obvious signs of tumor invasion. Case: We present a case of ovarian metastasis occurring in a 37-year-old triparous premenopausal woman with a diagnosed case of endocervical AIS. The patient presented with a rapidly growing and painful abdominal mass 22 months after a cold knife cone biopsy and 19 months after a simple extrafascial hysterectomy. She was subsequently treated with a combination of surgery and chemotherapy and is currently free of disease seven years after the initial diagnosis of endocervical AIS lacking unequivocally recognizable stromal invasion. Conclusion: Endocervical AIS with early or even no obvious detectable invasion may spread to the ovaries, but this situation is exceptionally rare. We propose that, in such cases, ovary sparing surgery is justifiable for initial treatment. Medical care strategies for patients affected by rare diseases largely depend on experience gained from reported cases in published literature, this is what compelled us to report this particular case.
\end{abstract}

Key words: Ovarian metastasis; Adenocarcinoma in situ; Uterine cervix.

\section{Introduction}

Cervical adenocarcinoma (AC) is increasing in worldwide incidence and currently accounts for more than $20 \%$ of all cervical cancers diagnosed $[1,2]$. Among all the superficially invasive cervical tumors, only $12 \%$ are early AC with the majority being squamous cell carcinoma (SCC) [1]. Several key differences exist between cervical AC and SCC including anatomic site of origin, risk factors, prognosis, pattern of dissemination, sites of recurrence, and rates of metastasis. Importantly, early AC has a favorable prognosis as the 5-year survival rate is greater than $80 \%$ [3]. The incidence of adenocarcinoma in situ (AIS) of the cervix has increased predominantly among nulliparous or primiparous reproductive-age women [4]. The distinction between early invasive AC and extensive AIS is usually subjective, and much more difficult to diagnose compared to differentiating early and superficially invasive SCC $[5,6]$. The optimal treatment for early invasive AC is controversial. Although curative therapy is pivotal, preservation of fertility is an important issue for reproductive-age patients and thus influences the choice of therapeutic strategy [7]. Such strategies range from radical hysterectomy with pelvic lymph node dissection, to cold knife cone biopsy of the cervix [7, 8]. Surgical treatment that preserves the ovaries benefits premenopausal women with cervical cancer [9].

Women with cervical AC are likely to have lymph node involvement, but not ovarian or hematogenous metastasis
[9]. However, some studies showed that ovarian metastases that simulate a primary ovarian tumor can occur in cases of early invasive cervical $\mathrm{AC}$ or even in cases that exhibit no obvious invasion [10]. Here we report a case of ovarian metastasis in a patient previously diagnosed with early invasive AC (FIGO IA1 stage tumor) of the cervix.

\section{Case Report}

A 37-year-old triparous premenopausal woman was referred to our Gynecological Oncology Department. Her PAP smear examination showed atypical glandular cells and cervical ectropion. She subsequently underwent colposcopy and cervical biopsy. Histopathological examination led to the identification of AIS. To this point, the patient had an unremarkable medical history, never took any hormonal replacement therapy, had no family history of carcinoma, and had a normal body mass index.

The patient subsequently underwent cold knife cone biopsy. Histological examination of numerous serial tissue sections (total of sixty sections) showed extensive AIS without obvious invasion. There were some foci suspicious for early invasion consisting of rare, single and small glandular structures in the stroma surrounded by prominent inflammatory infiltration. However, no unequivocally recognizable stromal invasion was seen in the majority of the AIS surrounding the stroma (Figure 1A). Tumor tissues showed strong and diffuse nuclear p16 immunoreactivity (Figure 1B). The excision margins of the cone were clear, and some
Eur. J. Gynaecol. Oncol. - ISSN: 0392-2936 XLI, n. 5, 2020

doi: $10.31083 /$ j.ejgo.2020.05.5155
This is an open access article under the CC BY 4.0 license (https://creativecommons.org/licenses/by/4.0/). 


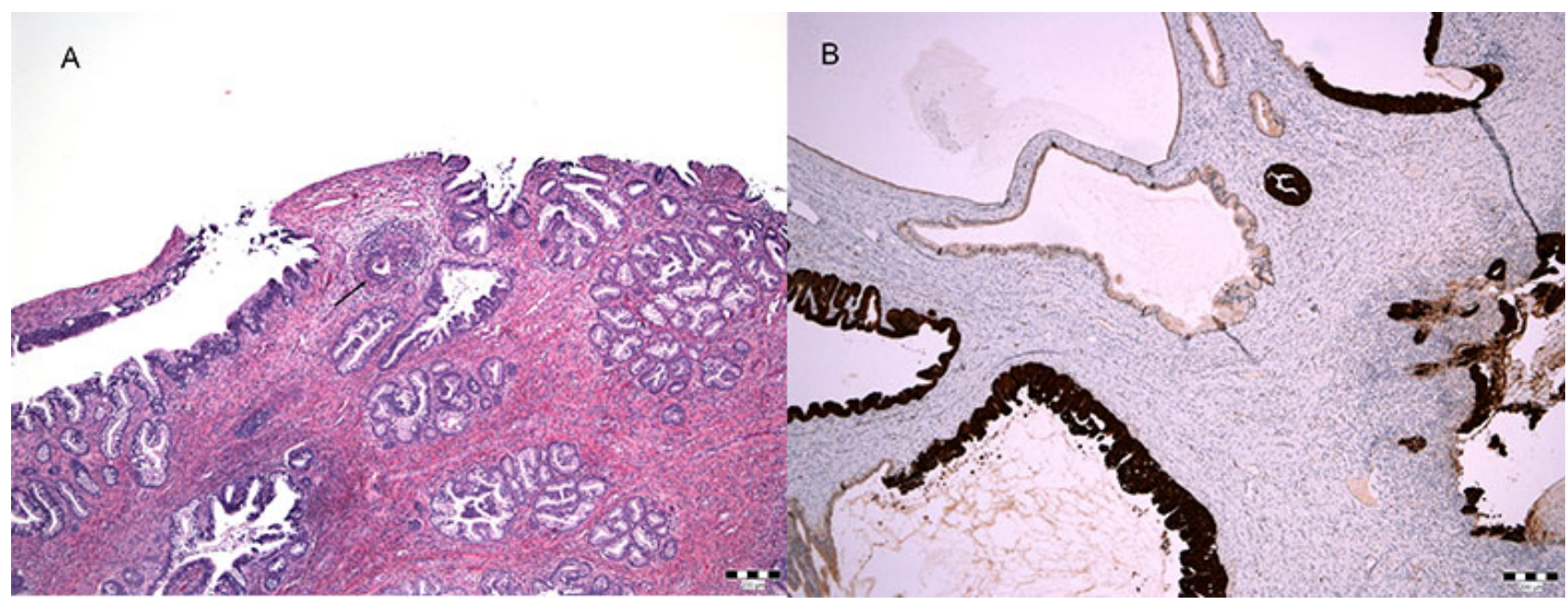

Figure 1. - A. Histological findings of the endocervical lesion. Extensive AIS with a focus suspicious for early invasion consisting of a small glandular structure in the stroma surrounded by more prominent inflammatory infiltration (arrow). No unequivocally recognizable stromal invasion was seen in the majority AIS surrounding stroma area. B. Tumor tissues showed strong and diffuse nuclear p16 immunoreactivity.

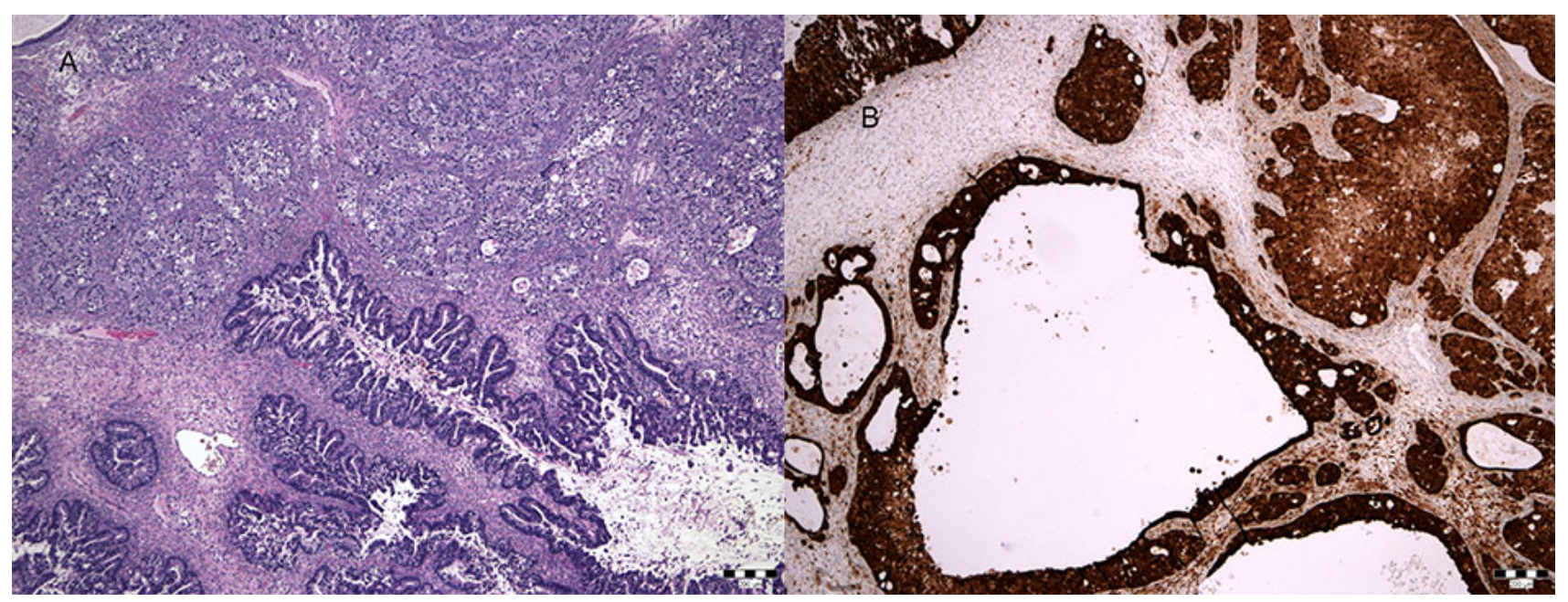

Figure 2. - A. Histological findings of the ovarian lesion. A similar morphology of the ovarian tumor was found when compared to the primary cervical AC (i.e., large areas of cystic glandular spaces lined by atypical epithelium showing marked mitotic and apoptotic activity), but there were also some solid areas with vacuolated and signet ring cells which were not present in the primary tumor of the cervix. B. Tumor cells showed diffuse and strong immunoreactivity for p16.

\section{AIS foci were visible close to the margin.}

This patient's case was presented at our tumor board meeting and simple hysterectomy was recommended. Laparoscopically assisted vaginal hysterectomy was performed three months after the cold knife cone biopsy. During intraoperative observation both ovaries were found macroscopically unremarkable, and the postoperative course was uneventful. Histological examination of the resected specimen revealed a small area of residual endocervical AIS with no obvious invasion. Endometrium and myometrium were unremarkable. The patient's case was presented at the postoperative multidisciplinary board meeting and no additional treatment was recommended. Five months after the operation her blood levels of CA 125, CA
15-3, CA 19-9 and CEA were examined and all values fell within normal ranges.

Nineteen months after the operation, the patient presented with a rapidly growing and painful abdominal mass. Ultrasonography showed an abdominal tumor with cystic and solid components occupying the pelvis and a portion of the left abdomen. CT scan revealed a left ovarian tumor of $10 \mathrm{~cm}$ in greatest diameter. Bilateral adnexectomy, omentectomy, multiple peritoneal biopsies, and lavage of the abdominal cavity for cytologic evaluation were subsequently performed. Pelvic and paraaortic lymph nodes were not palpably enlarged. There was no macroscopic residual tumor left in abdomen after the surgery. Intraoperative frozen section diagnosis of the left ovarian tumor was re- 
ported as ovarian carcinoma, probably primary mucinous carcinoma. Final histological examination revealed that the ovarian tumor had a similar morphology as the previously diagnosed primary cervical AC (i.e., there were large areas of cystic glandular spaces lined by atypical epithelium showing marked mitotic and apoptotic activity). However, in the ovarian tumor there were also solid areas with vacuolated and signet ring cells which were not present in the primary cervical tumor. The immunophenotype of the ovarian tumor was consistent with metastatic cervical AC with diffuse and strong positivities for p16, CK7, and CEA. Conversely, ER, PR and CK20 staining were completely negative. These findings led to the final diagnosis of cervical $\mathrm{AC}$ metastasis to the ovary (Figure $2 \mathrm{~A}$ and $2 \mathrm{~B}$ ).

Left fallopian tube, right adnexa, as well as samples of peritoneum and omentum taken at surgery were unremarkable. Cytologic evaluation of a lavage sample was negative for malignant cells. The postoperative course was uneventful. This case was presented at the postoperative multidisciplinary board meeting and adjuvant platinum-based chemotherapy with carboplatin and paclitaxel was recommended. Five months after the operation the patient completed six cycles of chemotherapy, and remains alive with no evidence of disease seven years after initial diagnosis of early invasive endocervical AC.

After treatment, a blood sample was sent out to examine genetic variations in the STK11 gene to rule out PeutzJeghers syndrome. The results were negative with only an unspecified variation with unknown impacts on protein function reported.

\section{Discussion}

Occurrence of synchronous or metachronous ovarian metastasis of early invasive cervical AC is a very rare event [11]. Without the use of ancillary techniques including HPV testing and/or immunohistochemistry for $\mathrm{p} 16$, the diagnosis is difficult because ovarian metastasis is often unilateral and morphologically similar to primary mucinous carcinoma or even borderline mucinous tumors [12]. When tumor is found in the ovary before or after the treatment of microinvasive cervical AC, it is generally thought to be a simultaneous emergence of a primary ovarian tumor. Further histological revision, immunohistochemistry for $\mathrm{p} 16$, and HPV testing may confirm metastasis from microinvasive $\mathrm{AC}$ within the ovary, and overrule diagnosis of primary ovarian tumor [12]. In this particular case, HPV testing was not carried out because at the time of initial AIS diagnosis the cancer lacked unequivocally recognizable stromal invasion and, accordingly, HPV testing was not yet an integral part of our routine management of this disease. However, subsequent positive immunohistochemical findings for $\mathrm{p} 16$ in both the primary cervical and metastatic tumor in the ovary supports the diagnosis of metastasis of HPV-related adenocarcinoma of the cervix to the ovary.

In the case presented in this report, the patient had endocervical microinvasive $\mathrm{AC}$ and the metastatic site within the ovary had an consistent pathological phenotype. The resected cervical specimen was extensively examined, and the patient was found to have no lymph node metastases. This leads us to speculate that the patient may have undergone a direct transuterine spread of neoplastic epithelium from the endocervix to the upper genital tract which was subsequently transported through the fallopian tubes to the ovary. This pattern of spread of cervical AC to the ovary $[12,13]$ is unusual and sometimes may resemble a primary ovarian neoplasm. One previous study [13] reported a significant correlation between corpus invasion and ovarian metastases of cervical AC, and suggested that tumors may locate at preferential lower uterine segment/corpus extension sites, particularly for minimally invasive tumors. This may represent an underappreciated risk factor for metastasis to the ovary, with retrograde uterine/transtubal spread as a possible mechanism of disease spread [13].

Because of the absence of established treatment schemes for this type of rare metastatic cervical AC, our patient received chemotherapy typically administered for treatment of a primary tumor of the ovary. Medical care strategies for patients affected by rare diseases largely depend on experiences gained from reported cases in literature, this is why we believe reporting this case is an important contribution.

Involvement of the ovaries by microinvasive $\mathrm{AC}$ represents a distinct pattern of spread in contrast to conventional metastasis to the ovary. Whereas metastasis generally occurs in advanced disease stages (i.e., FIGO IVA), the patient in this report had a favorable clinical outcome. These findings suggest that isolated metastases may not necessarily indicate a poor prognosis, as would be expected for advanced stage cervical $\mathrm{AC}$, and resection of the isolated metastasis may have therapeutic and survival benefits $[12,13]$. Additional follow-up will be required to determine the long-term prognosis for such patients [13].

In conclusion, although cervical AC with early or even questionable invasion may spread to the ovary, this is rare and we believe that an ovary sparing surgery is justifiable for initial treatment. The present case, as well as limited data available from the literature, suggests that the prognosis of these patients is relatively favorable. This perhaps stems from the mechanism of spread being different from that observed in more conventional cases of advanced cervical AC. More data are needed to establish the appropriate treatment scheme for cases meeting these criteria.

\section{Ethics approval and consent to participate}

Written informed consent was obtained from the patient for publication of this case report and accompanying images.

\section{Acknowledgements}

We thank Professor Glenn McCluggage, gynecological pathologist, from Department of Pathology from Belfast for consultation and review of the tumor. 


\section{Conflict of Interest}

The authors declare no conflict of interest.

Submitted: February 02, 2019

Accepted: April 09, 2019

Published: October 15, 2020

\section{References}

[1] Bray F.: "Incidence Trends of Adenocarcinoma of the Cervix in 13 European Countries". Cancer Epidemiol. Biomarkers Prev., 2005, 14, 2191-2199.

[2] Chan P.G., Sung H.-Y., Sawaya G.F.: "Changes in Cervical Cancer Incidence After Three Decades of Screening US Women Less Than 30 Years Old". Obstet. Gynecol., 2003, 102, 765-773.

[3] Quinn M.A., Benedet J.L., Odicino F., Maisonneuve P., Beller U., Creasman W.T., et al.: "Carcinoma of the Cervix Uteri". Int. J. Gynecol. Obstet., 2006, 95, S43-S103.

[4] Bullphelps S., Garner E., Walsh C., Gehrig P., Miller D., Schorge J.: "Fertility-sparing surgery in 101 women with adenocarcinoma in situ of the cervix". Gynecol. Oncol., 2007, 107, 316-319.

[5] Reynolds E.A., Tierney K., Keeney G.L., Felix J.C., Weaver A.L., Roman L.D., et al.: "Analysis of Outcomes of Microinvasive Adenocarcinoma of the Uterine Cervix by Treatment Type". Obstetr. Gynecol., 2010, 116, 1150-1157.

[6] Smrkolj Š., Pogačnik R.K., Slabe N., Rakar S.: "Clinical outcome of patients with FIGO stage IA2 squamous cell carcinoma of the uterine cervix". Gynecol.c Oncol., 2012, 124, 68-71.

[7] Touhami O., Plante M.: "Erratum to "Should ovaries be removed or not in (early-stage) adenocarcinoma of the uterine cervix: A review" [Gynecol. Oncol. 136 (2015) 384-388]". Gynecol. Oncol.,
2015, 137, 600 .

[8] Meglic L., Pogacnik R.K., Rakar S., Smrkolj S.J.E.J.o.G.O.: "Clinical outcome of patients with microinvasive adenocarcinoma of the uterine cervix". 2013, 34, 296-299.

[9] Shimada M., Kigawa J., Nishimura R., Yamaguchi S., Kuzuya K., Nakanishi T., et al.: "Ovarian metastasis in carcinoma of the uterine cervix". Gynecol. Oncol., 2006, 101, 234-237.

[10] Nakanishi T., Wakai K., Ishikawa H., Nawa A., Suzuki Y., Nakamura S., et al.: "A Comparison of Ovarian Metastasis between Squamous Cell Carcinoma and Adenocarcinoma of the Uterine Cervix". Gynecol. Oncol., 2001, 82, 504-509.

[11] Chang M.C., Nevadunsky N.S., Viswanathan A.N., Crum C.P., Feltmate C.M.: "Endocervical Adenocarcinoma In Situ With Ovarian Metastases: A Unique Variant With Potential for Long-term Survival”. Int. J. Gynecol. Pathol., 2010, 29, 88-92.

[12] Ronnett B.M., Yemelyanova A.V., Vang R., Gilks C.B., Miller D., Gravitt P.E., et al.: "Endocervical Adenocarcinomas With Ovarian Metastases". Am. J. Sur. Pathol., 2008, 32, 1835-1853.

[13] Tabata M., Ichinoe K., Sakuragi N., Shiina Y., Yamaguchi T., Mabuchi Y.: "Incidence of ovarian metastasis in patients with cancer of the uterine cervix". Gynecol. Oncol., 1987, 28, 255-261.

Corresponding Author:

ŠPELA SMRKOLJ, MD, Ph.D.

Division of Gynecology and Obstetrics

University Medical Centre Ljubljana

Slajmerjeva 3, SI-1000 Ljubljana

Slovenia (Europe)

E-mail: spela.smrkolj@mf.uni-lj.si

spsmrkolj@gmail.com 